LINHENG LI, Ph.D. Candidate ${ }^{1,2,3}$

E-mail: leelinheng@seu.edu.cn

JING GAN, Ph.D. Candidate ${ }^{1,2}$

E-mail: jinggan@seu.edu.cn

XU QU, Ph.D. ${ }^{1,2,3}$

(Corresponding author)

E-mail: quxu@seu.edu.cn

JIAN ZHANG, Ph.D. , $^{1,3}$

E-mail: jianzhang@seu.edu.cn

BIN RAN, Ph.D. . $^{1,2,3}$

E-mail: bran@seu.edu.cn

${ }^{1}$ School of Transportation, Southeast University

University Road \#2, Nanjing, 211189, China

2 Jiangsu Key Laboratory of Urban ITS

School of Transportation, Southeast University

Si Pai Lou \#2, 210096, Nanjing, China

${ }^{3}$ Institute on Internet of Mobility

Southeast University and University

of Wisconsin-Madison

2\# Si Pai Lou, 210096, Nanjing, China
Transport and Sustainable Development Original Scientific Paper Submitted: 20 Feb. 2020 Accepted: 4 June 2020

\title{
STABILITY AND ENVIRONMENTAL ANALYSIS OF MIXED TRAFFIC FLOW - USING THE MARKOV PROBABILISTIC THEORY
}

\section{ABSTRACT}

The rapid growth of $C A V$ (Connected and Automated Vehicle) market penetration highlights the need to gain insight into the overall stability of mixed traffic flows in order to better deploy CAVs. Several studies have examined the modelling process and stability analysis of traffic flow in a mixed traffic environment without considering its inner spatial distribution. In this paper, an innovative Markov chain-based model is established for integrating the spatial distribution of mixed traffic flow in the model process of car-following behaviour. Then the linear stability analysis of the mixed traffic flow is conducted for different CAV market penetration rates, different $C A V$ platoon strength and different cooperation efficiency between two continuous vehicles. Moreover, several simulations under open boundary conditions in multiple scenarios are performed to explicate how CAV market penetration rate, platoon strength and cooperation efficiency jointly influence the stability performance of the mixed traffic flow. The results reveal that the performance of this mixed traffic flow stability could be strengthened in these three factors. In addition to stability, an investigation of the fuel consumption and emission reduction under different market penetration rates and the platoon strength of CAVs are explored, suggesting that substantial potential fuel consumption and emission could be reduced under certain scenarios.

\section{KEY WORDS}

traffic flow modelling; linear stability; connected and automated vehicles; mixed traffic flow; Markov chain,

\section{INTRODUCTION}

In the Connected and Automated Vehicles (CAVs) system with the benefit of the advanced wireless communication technology, the CAVs can share real-time information with other vehicles and infrastructure [1]. The vehicles will accurately get information about the surrounding vehicles. Thus, the vehicles under the CAV environment tend to form a platoon-based driving pattern. In recent years, many scholars have carried out extensive research on platoon control of CAVs under advanced communications [2-5]. They point out that when the market penetration rate of CAVs is high enough, the CAV platoon has the potential to mitigate traffic congestion, increase road throughput, and reduce energy consumption through reasonable control. However, CAVs have not appeared on the market in large quantities, and they are only in the initial stage of the development. Thus, a mixed traffic environment, a combination of CAVs and human-driven vehicles (HVs), will continue for a relatively long 
time. Many findings have demonstrated that large amounts of CAVs are able to significantly enhance road traffic capacity, safety, fuel and emission efficiency [6-8]. And all these ameliorates are inseparable from the stability performance [9-13]. To better realize this expected potential boost provided by CAV to the traffic, the mixed traffic flow is desired to exhibit string-stable behaviour [14-18]. Many studies have been carried out for theoretically investigating the stability of the homogeneous traffic flow, which only contains HVs or CAVs, [3, 1922]. In addition, many scholars have also carried out theoretical analyses on the stability performance of various $\mathrm{CAV}$ market penetration under mixed traffic environment $[23,24]$. These studies have reached a consensus that traffic flow stability will improve with the increase of CAV market penetration rate.

Up to now, the impact of order randomness of the mixed traffic flow on the stability performance had not been apprehended in previous studies assuming deterministic time delays between two continuous vehicles. Ghiasi et al. put forward a concept of platoon strength; their findings proved that it will affect the capacity of mixed traffic to some extent; a Markov chain model which is proposed in their research is used to analytically formulate the traffic capacity of mixed traffic flow [25]. Although they mainly focus on the capacity analysis of mixed traffic flow, the method in this paper can be extended in a number of directions. Inspired by this, we believe that this Markov model can be applied to analyse the microscopic traffic dynamics of mixed traffic flow (e.g. the traffic flow stability analysis). The stability performance in the traffic flow is related to safety, throughput as well as energy, and the insights that reveal how CAV market penetration and platoon strength jointly influence the stability of the mixed traffic remain a challenge.

Furthermore, from the perspective of modelling, the IDM (Intelligent Driver Model) with or without time delay, describing the car-following behaviour is often used to analyse the string stability of traffic flow in traditional environment. Ngoduy uses an extension IDM with different time delays to obtain stability criterion for the mixed traffic system under connected and automated environment [14]. Li proposed another extended IDM in consideration of the cooperation efficiency between two continuous vehicles, but the authors assume that the cooperation efficiency is not related to the order of vehicles, and this assumption obviously does not accord with the actual situation [26]. In general, compared with the HVs, CAVs have stronger cooperation efficiency with its preceding vehicle. Thus the difference between different distribution types should be distinguished and investigated in this extended IDM. Therefore, this paper attempts to span the gap by proposing an innovative Markov chain-based model for the stability performance investigation under mixed traffic environment. This model will jointly take into account the effects of CAV market penetration, CAV platoon strength and collaboration efficiency between two adjacent vehicles.

Moreover, fuel consumption and emission reduction is another field where CAVs can make significant contributions [27-29]. This study also investigates how CAVs affect fuel consumption and emission from the perspective of stability analysis. The results reveal that the substantial potential energy saving and emission reduction could be achieved under certain scenarios.

The rest of this paper is organized as follows: Section 2 provides the modelling process of the proposed Markov chain-based model. Section 3 introduces string stability conditions for mixed traffic system in consideration of topology of vehicles, and Section 4 projects the effect of penetration rate, platoon strength of CAVs and collaboration efficiency into the stability analysis with different car-following simulation scenarios to support our analytical findings. In addition, fuel consumption and emission analysis obtained with the simulations are presented in Section 5. Finally, Section 6 concludes with some discussions and future research ideas.

\section{MODEL FORMULATION}

In this section, first, the spatial distribution randomness of mixed traffic flow is modelled. Additionally, the homogeneous traffic flow models of HVs and CAVs are briefly introduced. Finally, an innovative Markov chain-based model is constructed, which jointly take into consideration the CAV penetration, CAV platoon strength, as well as the collaboration efficiency between two adjacent vehicles.

\subsection{Spatial distribution model of mixed traffic flow}

In mixed traffic environment, the traffic flow will be randomly composed of CAVs and HVs. Markov chain describes a stochastic process in a state space 
that transitions from one state to another. The benefit from this character is that it could be well employed to depict the arrangement of mixed traffic flow problems as mentioned above.

For the sake of investigating the stochastic distribution of different vehicles, a Markov model raised by Ghiasi et al. [25] is employed to depict the stochastic distribution of the mixed traffic flow. Assume that there exist $N$ vehicles running on a certain road segment. $A_{n} \in S:\{0,1\}$ represents the state space variable of the $n$-th step, that is, at the $n$-th step, what type of vehicle there is, and the different values of $A_{n}$ represent different types of vehicles (if vehicle $n$ is an $\mathrm{HV}, A_{n}=0$; and if vehicle $n$ is a CAV, $A_{n}=1$ ), and let $p_{1}$ represent the CAV market penetration, then we have:

$p_{1}=\sum_{n=1}^{N} \frac{A_{n}}{N}$

Since the traffic flow is composed of only two vehicle classes (CAV and $\mathrm{HV}$ ), the market penetration of $\mathrm{HV} p_{0}$ can be obtained as:

$p_{0}=\sum_{n=1}^{N} \frac{1-A_{n}}{N}=1-p_{1}$

Because $p_{1}$ and $p_{0}$ exist, the relationship is based on the formulations of Equation 2. So, in the following paper, only $p_{1}$ will be used for the analysis. Let us assume that the probability of the first vehicle being a $\mathrm{CAV}$ is $p_{1}$, that is, $\operatorname{Pr}\left(A_{1}=1\right)=p_{1}$. Hence, the initial state of the system $S_{\text {initial }}$ and the subsequent transition matrix can be represented as indicated in literature [25]:

$$
\begin{aligned}
& S_{\text {initial }}:=\left\{p_{1}, p_{0}\right\} \\
& T_{\text {trans }}:=\left[\begin{array}{ll}
T_{11} & T_{10} \\
T_{01} & T_{00}
\end{array}\right]
\end{aligned}
$$

where $S_{\text {initial }}$ denotes the initial state of the system; and $T_{i j}$ represents the probability for different car-following scenarios, e.g. $T_{01}:=\operatorname{Pr}\left(A_{n+1}=C A V\right.$ $\left.A_{n}=H V\right)$.

Then, $T_{i j}$ could be expressed as a function of $p_{1}$ and $O$ :

$$
\begin{aligned}
& \left\{\begin{array}{l}
T_{10}\left(p_{1}, O\right)=\left(1-p_{1}\right)(1-O) \\
T_{11}\left(p_{1}, O\right)=1-T_{10}\left(p_{1}, O\right)
\end{array}\right. \\
& \left\{\begin{array}{l}
T_{01}\left(p_{1}, O\right)=p_{1}(1-O) \\
T_{00}\left(p_{1}, O\right)=1-T_{01}\left(p_{1}, O\right)
\end{array}\right.
\end{aligned}
$$

where $O \in[0,1]$ denotes the connection degree of the same type of vehicles, with the increase of $O$. It is indicated that the two vehicles of the same type are more likely to appear adjacent to each other. When $O=1$, the same type of vehicles will be adjacent to each other, at this time, the platoon composed of CAVs has the largest capacity, as shown in Figure 1. Otherwise, if $O \in[0,1)$, HVs and CAVs will be randomly distributed, as shown in Figure 2. In particular, the distribution of vehicles will only be determined by CAV market penetration when $O=0$.

\subsection{Homogeneous car-following models}

The IDM is adopted to describe the behaviour of HVs. And the model derived by the PATH program is employed to depict the car-following behaviour of CAVs.

\section{Modelling for human-driven vehicles}

IDM was proposed by Treiber et al. in 2000 . This model has the following expression:

$$
\begin{aligned}
a_{n}(t) & =\frac{d v_{n}(t)}{d t}=f_{n}\left(s_{n}(t), v_{n}(t), \Delta v_{n}(t)\right)= \\
& =\alpha\left[1-\left(\frac{v_{n}(t)}{v_{0}}\right)^{4}-\left(\frac{s_{e}^{*}}{s_{n}(t)}\right)^{2}\right]
\end{aligned}
$$

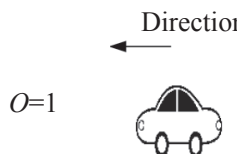

HV

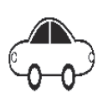

$\mathrm{HV}$

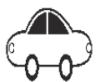

$\mathrm{HV}$

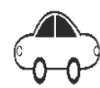

$\mathrm{HV}$

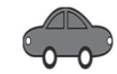

CAV

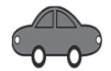

CAV

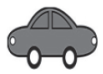

CAV

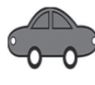

CAV

Figure 1 -Vehicle distribution example when $O=1$

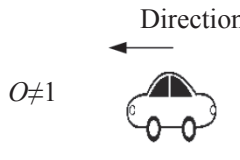

HV
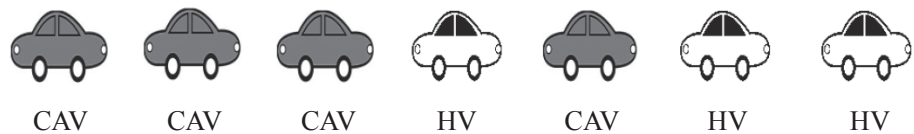

HV

HV

Figure 2 - Vehicle distribution example when $O \neq 1$ 
where

$$
s_{e}^{*}=s_{0}+v_{n}(t) T+\frac{v_{n}(t) \Delta v_{n}(t)}{2 \sqrt{\alpha \beta}}
$$

In this model, $a_{n}(t)$ is the acceleration of $n$-th vehicle at the next time step; $v_{n}(t)$ denotes current velocity of $n$-th vehicle; $s_{n}(t)$ is the headway between two vehicles; $\Delta v_{n}(t)$ represents the velocity difference between two car-following vehicles.

The description of $\alpha, v_{0}, s_{0}, T, \beta$ are as follows:

$\alpha$ - vehicle maximum acceleration;

$v_{0}$ - expected velocity at which vehicles drive in free state;

$s_{e}^{*}-$ desired safe headway;

$s_{0}$ - minimum desired distance. If the headway between two adjacent vehicles is less than $s_{0}$, the vehicle will stop going

$T$ - minimum possible time that takes for trailing the preceding vehicle to cover the distance;

$\beta-$ desired deceleration, which is a positive number.

Based on Equations 6 and 7. The three partial derivatives of this model regarding $v_{n}, \Delta v_{n}, s_{n}$ at steady state can be calculated through Equation 8:

$$
\left\{\begin{array}{l}
f_{n}^{H v}=\left.\frac{\partial f_{n}}{\partial v_{n}}\right|_{\left(v_{e}, s_{e}\right)}=-2 \alpha\left[\frac{2}{v_{0}}\left(\frac{v_{e}}{v_{0}}\right)^{3}+\frac{T\left(s_{0}+T v_{e}\right)}{\left(s_{e}\right)^{2}}\right] \\
f_{n}^{H \Delta v}=\left.\frac{\partial f_{n}}{\partial \Delta v_{n}}\right|_{\left(v_{e}, s_{e}\right)}=-\frac{v_{e}}{S_{e}} \sqrt{\frac{\alpha}{\beta}} \frac{S_{0}+T v_{e}}{S_{e}} \\
f_{n}^{H S}=\left.\frac{\partial f_{n}}{\partial s_{n}}\right|_{\left(v_{e}, s_{e}\right)}=\frac{2 \alpha}{S_{e}}\left(\frac{s_{0}+T v_{e}}{S_{e}}\right)^{2}
\end{array}\right.
$$

where $f_{n}^{H}$ is human-driven vehicle acceleration function, where $H$ stands for HV. In addition, $f_{n}^{H}$, $f_{n}^{H \Delta v}$ and $f_{n}^{H S}$ denote the partial differential of $f_{n}$ with respect to velocity, velocity difference, and headway distance at equilibrium state, respectively.

\section{Modelling CAVS}

In the research of Milanés and Shladover [31], PATH program put forward one car-following model for CAVs, which has been widely employed by many scholars. The acceleration of $n$-th $\mathrm{CAV}$ at time $t$ is depicted by:

$$
a_{n}=\frac{d v_{n}(t)}{d t}=\frac{k_{1}\left(s_{n}(t)-s_{0}-t_{c} v_{n}\right)+k_{2} \Delta v_{n}}{\Delta t+k_{2} t_{c}}
$$

where $s_{0}$ has the same meaning as described in Equation $7 ; t_{c}$ indicates the expected time gap of CAVs; $k_{1}$ and $k_{2}$ are parameters which need to be calibrated; and $\Delta t$ is the time step. Some experiments were performed by PATH to calibrate these parameters whose values are listed in Table 3 of Section 4.

The three partial differentials of this model regarding $v_{n}, \Delta v_{n}, s_{n}$ at steady state can be calculated as the following formulas on the basis of Equation 9:

$$
\left\{\begin{array}{l}
f_{n}^{C v}=\left.\frac{\partial f_{n}}{\partial v_{n}}\right|_{\left(v_{e}, s_{e}\right)}=-\frac{k_{1} t_{c}}{\Delta t+k_{2} t_{c}} \\
f_{n}^{C \Delta v}=\left.\frac{\partial f_{n}}{\partial \Delta v_{n}}\right|_{\left(v_{e}, s_{e}\right)}=\frac{k_{2}}{\Delta t+k_{2} t_{c}} \\
f_{n}^{C S}=\left.\frac{\partial f_{n}}{\partial s_{n}}\right|_{\left(v_{e}, s_{e}\right)}=\frac{k_{1}}{\Delta t+k_{2} t_{c}}
\end{array}\right.
$$

\subsection{Markov Chain-based model}

In CAV environment, the information sharing efficiency depends in part on the collaboration efficiency between two adjacent vehicles. This study employs the refined model put forward by Li et al. [26] to depict CAV car-following behaviour, due to its refined consideration of both time delay and collaboration efficiency with its preceding vehicle. The driving behaviour of CAVs is described as:

$$
\begin{aligned}
a_{n} & =\frac{d v_{n}\left(t+t_{d}\right)}{d t}=f_{n}\left(s_{n}(t), v_{n}(t), \Delta v_{n}(t)\right)+ \\
& +\lambda f_{n-1}\left(s_{n-1}(t), v_{n-1}(t), \Delta v_{n-1}(t)\right)
\end{aligned}
$$

where $t_{d}$ indicates the delay due to physical effects of the vehicles; $\lambda$ denotes collaboration efficiency between two adjacent vehicles, as depicted in Figure 3.

Comparing to the IDM and PATH model that considers only speed, headway, and relative speed, this model depicts that collaboration efficiency is another factor that affects the driving behaviour of the vehicle. Based on such a model, a novel Markov chain-based model that premeditates different collaboration efficiencies between two adjacent vehicles could be easily explored.

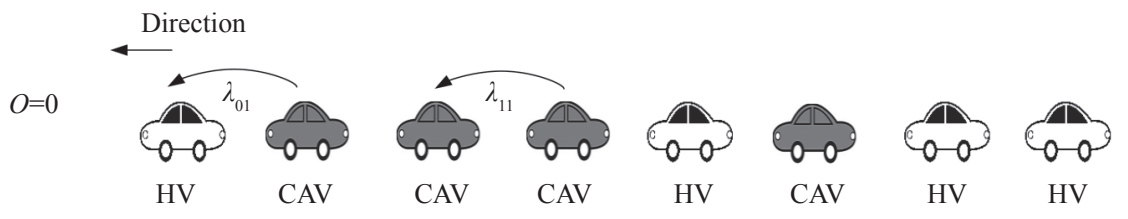

Figure 3 -Different collaboration efficiencies for two adjacent vehicles when $O \neq 1$ 
To explore how CAV market penetration, collaboration efficiency and CAV platoon strength affect the traffic flow stability, an innovative Markov chain-based model that incorporates these three key factors is proposed in this section.

In more general cases of $O \in[0,1), \mathrm{CAV}$ s respond faster to the state changes in the preceding vehicle in comparison with humans, benefiting from the wireless communication with vehicles and infrastructure. As HV cannot sensor the condition of the preceding vehicle without on-board equipment, it can only pay attention to the headway with its preceding vehicle, and therefore the decision of HV is independent of the type of its preceding vehicle. Thus, $\lambda$ is variable rather than fixed, which depends on the types of two adjacent vehicles. Figure 3 depicts two different scenarios of collaboration efficiency $\lambda_{01}$ and $\lambda_{11}$, which denote the car-following scenarios that an HV or a CAV follows a CAV, respectively.

Therefore, different probabilities of $n$-th vehicle acceleration under different car-following circumstances can be obtained:

Table 1 - Probability of $n$-th vehicle acceleration

\begin{tabular}{|c|c|}
\hline Car-following scenarios & Probability \\
\hline HV (front) + CAV (rear) & $P_{0} T_{01} f_{n}^{C+}+\lambda_{01} P_{0}^{2} T_{01} f_{n-1}^{H}$ \\
\hline HV (front) + HV (rear) & $P_{0} T_{00} f_{n}^{H}$ \\
\hline CAV (front) + HV (rear) & $P_{1} T_{10} f_{n}^{H}$ \\
\hline CAV (front) + CAV (rear) & $P_{1} T_{1} f_{n}^{C+}+\lambda_{11} P_{1}^{2} T_{11} f_{n-1}^{C}$ \\
\hline
\end{tabular}

Based on the analysis above, the acceleration of the $n$-th vehicle could be thus presented as:

$$
\begin{aligned}
& \frac{d v_{n}\left(t+t_{d}\right)}{d t}=\left[\left(1-p_{1}\right) T_{00}+p_{1} T_{10}\right] f_{n}^{H}+ \\
& \quad+\left(1-p_{1}\right)^{2} \lambda_{01} T_{01} f_{n-1}^{H}+ \\
& \quad+\left[\left(1-p_{1}\right) T_{01}+p_{1} T_{11}\right] f_{n}^{C}+p_{1}^{2} \lambda_{11} f_{n-1}^{C}
\end{aligned}
$$

Substituting Equation 5 into Equation 12, the following equation can be derived:

$$
\begin{aligned}
& \frac{d v_{n}\left(t+t_{d}\right)}{d t}=\left(1-p_{1}\right) f_{n}^{H}+\left[p_{1}\left(1-p_{1}\right)^{2}(1-O)\right] \lambda_{01} f_{n-1}^{H} \\
& \quad+p_{1} f_{n}^{C}+p_{1}^{2}\left(O+p_{1}-p_{1} O\right) \lambda_{11} f_{n-1}^{C}
\end{aligned}
$$

For notation convenience, let $A_{1}, A_{2}, A_{3}, A_{4}$ denote the four coefficients in Equation 13, respectively:

$$
\begin{aligned}
& A_{1}=1-p_{1} \\
& A_{2}=p_{1}\left(1-p_{1}\right)^{2}(1-O) \lambda_{01} \\
& A_{3}=p_{1} \\
& A_{4}=p_{1}^{2}\left(O+p_{1}-p_{1} O\right) \lambda_{11}
\end{aligned}
$$

Then Equation 13 can be simplified to: $\frac{d v_{n}\left(t+t_{d}\right)}{d t}=A_{1} f_{n}^{H}+A_{2} f_{n-1}^{H}+A_{3} f_{n}^{C}+A_{4} f_{n-1}^{C}$

Note that Equation 6 and Equation 11 can be retrieved if a homogeneous traffic flow environment is presented.

\section{LINEAR STABILITY ANALYSIS}

\subsection{Mathematical derivation of stability conditions}

The general control theory is employed to derive the stability criterion for our innovative Markov chain-based model. The traffic flow stability is affected by many factors, and traffic disturbance is one of the important ones. Let $y_{n}(t)$ be one small perturbation of $n$-th vehicle at time $t$ :

$y_{n}(t)=C e^{i a k n+z t}=x_{n}(t)-\bar{x}_{n}(t)$

where $C$ is a constant and $a_{k}=2 \pi k / N, k=0,1, \ldots N-1$.

Performing the second derivative on both sides of Equation 16, the following equation can be derived:

$$
\begin{aligned}
& \ddot{y}_{n}\left(t+t_{d}\right)=\ddot{x}_{n}\left(t+t_{d}\right)-\left(\bar{x}_{n}\left(t+t_{d}\right)\right)^{n}=\ddot{x}_{n}\left(t+t_{d}\right)= \\
& \quad=\frac{d v_{n}\left(t+t_{d}\right)}{d t}
\end{aligned}
$$

Substituting Equation 15 into Equation 17, one can obtain:

$$
\ddot{y}_{n}\left(t+t_{d}\right)=A_{1} f_{n}^{H}+A_{2} f_{n-1}^{H}+A_{3} f_{n}^{C}+A_{4} f_{n-1}^{C}
$$

By linearizing Equation 18, multiplying $t_{d}$ on both sides, and submitting $y_{n}(t)=C e^{i a_{k} n+z t}$ and $\dot{y}_{n}(t)=z C e^{i a k n+z t}$, we can get the following equation:

$$
\begin{aligned}
& \left(e^{t d z}-1\right)\left[z e^{t d z}-\left(A_{1} f_{n}^{H v}+A_{3} f_{n}^{C v}\right)-\left(A_{2} f_{n-1}^{H v}+A_{4} f_{n-1}^{C v}\right) e^{-i a k}-\right. \\
& \left.-\left(A_{1} f_{n}^{H \Delta v}+A_{3} f_{n}^{C \Delta v}\right)\left(1-e^{-i a k}\right)-\left(A_{2} f_{n-1}^{H \Delta v}+A_{4} f_{n-1}^{C \Delta v}\right)\left(e^{-i a_{k}}-e^{-2 i a_{k}}\right)\right] \\
& =t_{d}\left[\left(A_{1} f_{n}^{H S}+A_{3} f_{n}^{C S}\right)\left(e^{-i a_{k}}-1\right)+\left(A_{2} f_{n-1}^{H S}+A_{4} f_{n-1}^{C S}\right)\left(e^{-2 i a_{k}}-e^{-i a_{k}}\right)\right]
\end{aligned}
$$

where the six partial derivatives are presented in Equations 8 and 10, respectively.

The solutions for $z$ can be obtained by substituting the expansion formulation $z=z_{1}\left(i a_{k}\right)+$ $z_{2}\left(i a_{k}\right)^{2}+\ldots, e^{t d z}=1+t_{d} z+t_{d}^{2} z^{2} / 2+\ldots$, and $e^{-n i a_{k}=1-i a_{k}}$ $n^{2} a_{k}^{2} / 2+\ldots$ into Equation 19 as discussed in [32]:

$z_{1}=\frac{A_{1} f_{n}^{H S}+A_{2} f_{n-1}^{H S}+A_{3} f_{n}^{C S}+A_{4} f_{n-1}^{C S}}{A_{1} f_{n}^{H v}+A_{2} f_{n-1}^{H v}+A_{3} f_{n}^{C v}+A_{4} f_{n-1}^{C v}}$

$$
\begin{aligned}
& z_{2}=\frac{\left[1-\frac{1}{2} t d\left(A_{1} f_{n}^{H v}+A_{2} f_{n-1}^{H v}+A_{3} f_{n}^{C v}+A_{4} f_{n-1}^{C v}\right)\right] z_{1}^{2}}{A_{1} f_{n}^{H v}+A_{2} f_{n-1}^{H v}+A_{3} f_{n}^{C v}+A_{4} f_{n-1}^{C v}}+ \\
& +\frac{\left(A_{2} f_{n-1}^{H v}+A_{4} f_{n-1}^{C v}-A_{1} f_{n}^{H \Delta v}-A_{2} f_{n-1}^{H \Delta v}-A_{3} f_{n}^{C \Delta v}-A_{4} f_{n-1}^{C \Delta v}\right)_{1}}{A_{1} f_{n}^{H v}+A_{2} f_{n-1}^{H v}+A_{3} f_{n}^{C v}+A_{4} f_{n-1}^{C v}}+ \\
& +\frac{\frac{1}{2} A_{1} f_{n}^{H S}+\frac{3}{2} A_{2} f_{n-1}^{H S}+\frac{1}{2} A_{3} f_{n}^{C S}+\frac{3}{2} A_{4} f_{n-1}^{C S}}{A_{1} f_{n}^{H v}+A_{2} f_{n-1}^{H v}+A_{3} f_{n}^{C v}+A_{4} f_{n-1}^{C v}}
\end{aligned}
$$


The traffic flow will keep in a steady state if $z_{2}>0$. Additionally, the following equations need to be satisfied in the case that the traffic flow consists of only one type of vehicle (pure CAV or pure $\mathrm{HV}): \quad f_{n}^{H v}=f_{n-1}^{H \nu} ; \quad f_{n}^{C v}=f_{n-1}^{C v} ; f_{n}^{H S}=f_{n-1}^{H S} ; f_{n}^{C S}=f_{n-1}^{C S}$; $f_{n}^{H \Delta v}=f_{n-1}^{H \Delta v} ; f_{n}^{C \Delta v}=f_{n-1}^{C \Delta v}$. Therefore, the stability criterion of a mixed traffic system can be derived as the following inequality:

$$
\begin{aligned}
& \frac{1}{2} t_{d}<\frac{1}{\left(A_{1}+A_{2}\right) f_{n}^{H v}+\left(A_{3}+A_{4}\right) f_{n}^{C v}-} \\
& -\frac{\left(A_{1}+A_{2}\right) f_{n}^{H \Delta v}+\left(A_{3}+A_{4}\right) f_{n}^{\Delta v}-A_{2} f_{n}^{H v}-A_{4} f_{n}^{C v}}{\left(A_{1}+A_{2}\right) f_{n}^{H s}+\left(A_{3}+A_{4}\right) f_{n}^{C s}}- \\
& -\frac{\left[\left(\frac{1}{2} A_{1}+\frac{3}{2} A_{2}\right) f_{n}^{H S}+\left(\frac{1}{2} A_{3}+\frac{3}{2} A_{4}\right) f_{n}^{C s}\right]\left[\left(A_{1}+A_{2}\right) f_{n}^{H v}+\left(A_{3}+A_{4}\right) f_{n}^{C v}\right]}{\left[\left(A_{1}+A_{2}\right) f_{n}^{H s}+\left(A_{3}+A_{4}\right) f_{n}^{S}\right]^{2}}
\end{aligned}
$$

where the values of coefficients $A_{1}, A_{2}, A_{3}, A_{4}$ are as shown in Equation 14.

Note that the stability criterion of IDM in [15] can be retrieved if a homogeneous traffic flow environment is presented and collaboration efficiency is equal to zero.

\subsection{Numerical analysis}

As shown in Inequality 22 and Equation 15, there are three free parameters that control the stability of the system: CAV market penetration rate $p_{1}, \mathrm{CAV}$ platoon strength $O$, and collaboration efficiency between two adjacent vehicles $\lambda_{01}, \lambda_{11}$. For the sake of gaining deep insights into the contributions of these critical factors to stability, some numerical analyses are conducted in this section. According to some recommendations of previous literature, the value of some parameters for our proposed Markov Chain-based model is presented in Table 2. In addition, the sensitivity analysis of model parameters is shown in Figures 4-7.

Figure 4 illustrates the stability boundary under different values of market penetration rate and platoon strength of CAVs in velocity and desired time

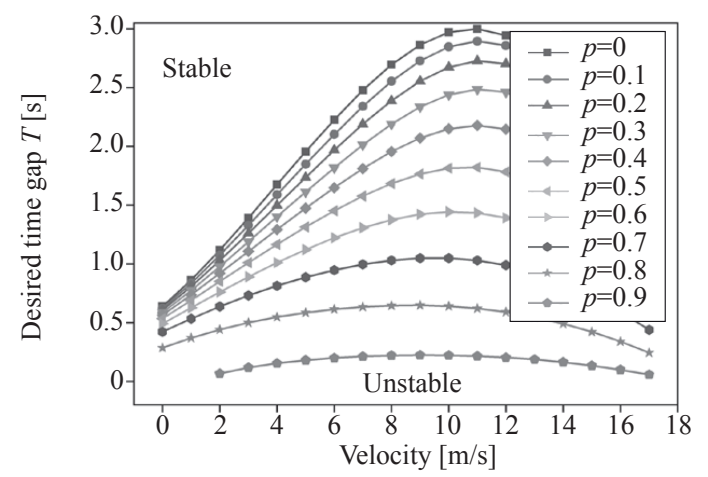

a) Market penetration gap plane, with $\lambda_{01}=0.3, \lambda_{11}=0.5$ and $t_{d}=0$. These marginal stability curves lie between two regions; the upper area of the edge stability curve represents the stable area, and the lower area denotes the unstable one. From Figure $4 a$ it is obvious that the position of the curve will be closer to x-axis when market penetration $p_{1}$ increases, which means that the unstable area will shrunk with the augment of $p_{1}$. The contribution of market penetration to stability performance is relatively minor at low market shares, resulting from the advantage of CAV that cannot be reflected, i.e. shorter reaction time, higher collaboration efficiency. On the other hand, CAVs make a greater contribution to stability as the market penetration rate increases, particularly under the circumstance where traffic flow is mainly composed of CAVs. Moreover, under equal market penetration circumstance, the biggest platoon strength can make more contributions than the smallest one in terms of enhancing stability performance, as shown in Figure $4 b$.

Furthermore, the gap between two adjacent curves becomes larger as CAVs market penetration increases. This is because the probability of a CAV following another CAV is $p_{1}^{2}$ when $O=0$ and $p_{1}$ when $O=1$. Thus, the system will be much more stable with high market penetration $p_{1}$. This is also the reason why stability performance is better when $O=1$ than $O=0$, as shown in Figure $4 b$.

To capture how collaboration efficiency affects the stability performance, the stability performance results for mixed traffic flow with various collaboration efficiencies are shown in Figure 5. The right hand of each subfigure illustrates the stability of contour plots, the red part (positive section) indicates the stability of the system, while the other parts (negative section) represent instability of the system.

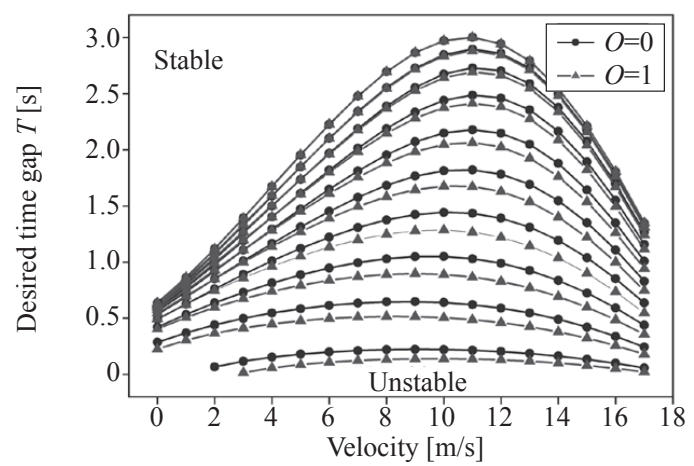

b) Platoon strength of CAVs on traffic stabilities

Figure 4-Stability boundary 

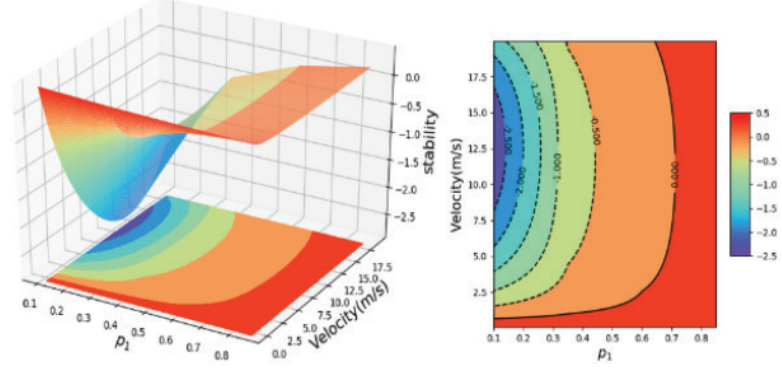

a) $t_{d}=0, O=1, \lambda_{01}=0, \lambda_{11}=0$

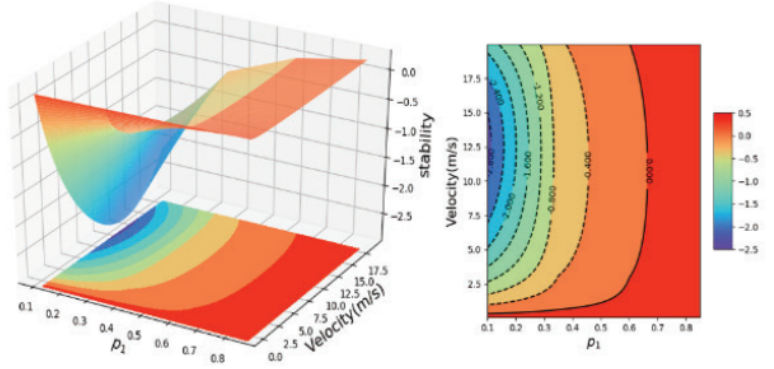

c) $t_{d}=0.5, O=1, \lambda_{01}=0.1, \lambda_{11}=0.8$

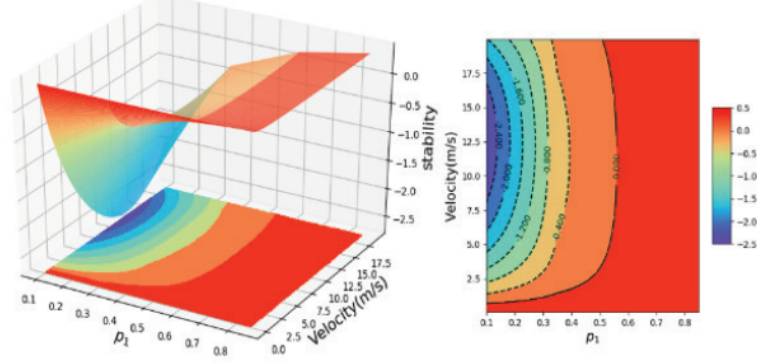

b) $t_{d}=0, O=1, \lambda_{01}=0.3, \lambda_{11}=0.5$
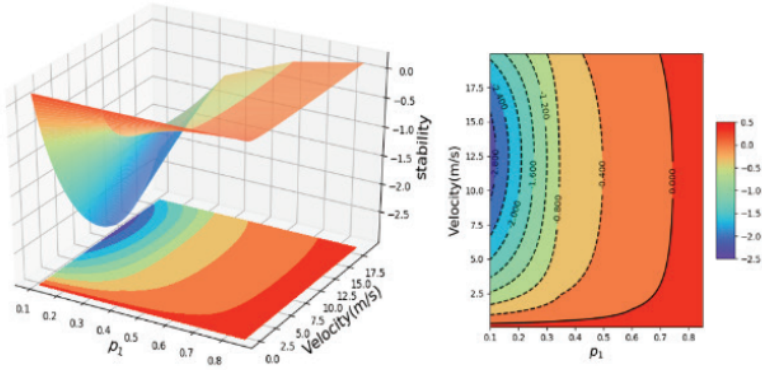

d) $t_{d}=0.5, O=1, \lambda_{01}=0.3, \lambda_{11}=0.5$

Figure 5 - Stability criterion under different combinations of CAV market penetration and collaboration efficiency with $t_{d}=0$

Moreover, comparing Figure $5 a$ with Figure $5 b$ and comparing Figure $5 \mathrm{c}$ with Figure $5 d$, it can be seen that as collaboration efficiency improves, the system stability can be greatly increased. However, the distribution of the market penetration rate to stability performance remains dominant. These numerical analysis results also indicate that as CAV market share increases, the stability performance and the critical speed are simultaneously improved.

In addition, physical delay $t_{d}$ can also affect stability performance, as shown in Figures $5 c$ and $5 d$. It can be easily found that shorter physical delay will make the system more stable. This conclusion is in consistence with the finding of literature $\mathrm{Li}$ et al. [26]. More specifically, comparing Figure $5 b$ with Figures $5 c$ and $5 d$, one can see that the physical delay has a more significant impact on system stability than collaboration efficiency, thus reducing physical delay is of great importance for the mixed traffic flow to exhibit string-stable behaviour.

\section{SIMULATION AND DISCUSSION}

In this section, several simulations are conducted for convincing the analysis of our analytical results. All of the simulations in this section are conducted on a single line freeway. There are 25 vehicles mixed by CAVs and some HVs on this freeway without overtaking and lane-change. Let them have the same initial headway $h=20 \mathrm{~m}$ and the same steady velocity $v_{0}=20 \mathrm{~m} / \mathrm{s}$. That is, the system is set in an equilibrium status at the beginning of the simulation. These vehicles including HVs and CAVs are distributed randomly along one segment of the freeway and numbered sequentially from 0 to 24 .

In order to analyse the stability performance based on our proposed Markov chain-based model, a small perturbation generated by the leading vehicle in the initial stable status is created. In this paper, traffic perturbation is conducted by suddenly decelerating the first vehicle of the fleet from $20 \mathrm{~m} / \mathrm{s}$ to $18 \mathrm{~m} / \mathrm{s}$ after the simulation running for $100 \mathrm{~s}$. The following vehicles will respond to this action by changing their status according to Equation 13 and the leading vehicle will maintain its velocity of $18 \mathrm{~m} / \mathrm{s}$ in the remaining simulation time.

To investigate the influence of CAV market penetration and the platoon strength on the stability performance, several simulations are conducted by changing the values of these two factors.

The first set of simulations examine the impacts of market penetration on the stability performance. Figure 6 compares the perturbation propagation along with the mixed traffic flow by changing six various CAV market penetrations, i.e. $p_{1}=0.1,0.3,0.5,0.6,0.9,1$, respectively, with parameter values of the driver model in Table 2. It is apparent from this figure that the stability of the system will be strengthened with the augment of 
Table 2 - Parameter values of IDM for HVs and PATH for CAVS

\begin{tabular}{|c|c|c|c||}
\hline $\begin{array}{c}\text { IDM } \\
\text { Parameter }\end{array}$ & Value for HVs & $\begin{array}{c}\text { PATH } \\
\text { Parameter }\end{array}$ & $\begin{array}{c}\text { Value for } \\
\text { CAVs }\end{array}$ \\
\hline \hline$T$ & $2.0 \mathrm{~s}$ & $k_{1}$ & 0.45 \\
\hline$s_{0}$ & $2.0 \mathrm{~m}$ & $k_{2}$ & 0.25 \\
\hline$a$ & $1.0 \mathrm{~m} / \mathrm{s}^{2}$ & $t_{c}$ & 0.6 \\
\hline$b$ & $2.0 \mathrm{~m} / \mathrm{s}^{2}$ & $\Delta t$ & 0.1 \\
\hline$v_{0}$ & $20 \mathrm{~m} / \mathrm{s}^{2}$ & & \\
\hline
\end{tabular}

Table 3 -Other parameters and their values for the simulation

\begin{tabular}{||c|c|c|c|c||}
\hline \multirow{2}{*}{ Variable } & \multirow{2}{*}{$\begin{array}{c}\text { Platoon } \\
\text { strength } \\
O\end{array}$} & \multirow{2}{*}{$\begin{array}{c}\text { Physical } \\
\text { delay } t_{d}\end{array}$} & \multicolumn{2}{|c|}{$\begin{array}{c}\text { Collaboration } \\
\text { efficiency }\end{array}$} \\
\cline { 4 - 5 } & & & $\lambda_{01}$ & $\lambda_{11}$ \\
\hline \hline Value & 1 & 0 & 0.3 & 0.5 \\
\hline
\end{tabular}

$p_{1}$, which is found in good agreement with the numerical investigation in Section 3. Although the system stability is strengthened as $p_{1}$ increases, the traffic system is still in an unstable condition until CAV penetration rate exceeds 0.9 , because the stability criterion Equation 22 cannot be satisfied with parameter values in Table 3.

Additionally, some other meaningful simulations under different value of platoon strength $O$ with same $p_{1}$ are conducted to explore how these two critical factors jointly influence the stability of a mixed traffic system. Figure 7 illustrates the speed profiles over time of the $2^{\text {nd }}, 5^{\text {th }}$ and $10^{\text {th }}$ vehicle under different combination of platoon strength $O$ and market penetration rate $p_{1}$, e.g. $p=0.1, O=0$; $P=0.1, O=1$. It could be found that the magnitude of velocity when $O=1$ is less than that in the case of $O=0$ until $p_{1}>0.3$, which indicates that the stability will not be strengthened but exacerbates in the case of a relatively low CAV market penetration rate (compare Figures $7 a$ and $7 b$ with Figures $7 c$ and $7 d$ ). The reason is that CAVs have excellent ability to absorb disturbance, which has a particularly large advantage in improving the system stability. Thus, the stability will be significantly strengthened as CAV market penetration augments. In our simulation case, if CAV market share is relatively low $\left(p_{1}=0.1\right)$, CAV platoon cannot completely absorb the disturbance at one time even if all the CAVs are platooned together. Therefore, the disturbance will propagate and grow in the remaining
HVs. On the contrary, if we spread all the CAVs apart in this case, the ability to absorb disturbances of every CAV will be better exerted. Every time the disturbance propagates, it will be filtered by the scattered CAVs, which can better improve the stability performance. In general, the improvement of the stability of traffic flow helps to improve the road capacity. This analytical analysis result is consistent with the conclusion derived in [33], in contrast with most people's intuitive feeling that higher market penetration and platoon strength are always beneficial to all aspects of the overall transportation system. In fact, these two factors may hinder the improvement of capacity in some cases. It is worth noting that in the case of other simulations, different conclusions may be drawn. But anyway, our conclusion can be a good counter-example for most people's intuitive feeling that higher platoon intensities always produce better stability performance.

Moreover, one should not ignore that the influence of platoon strength could be considered as negligible in the case of high CAV market penetration. In other words, market penetration is more effective in improving the stability performance of the traffic system than the platoon strength, no matter whether at low market penetration rate or high rate. When $p_{1}>0.3$, with the increase of CAV platoon strength, CAVs are more likely to run in the form of the platoon in which the headway of two consecutive vehicles is much shorter than two conventional HVs. This is why platoon strength is of benefit to enhance the stability performance after penetration rate reaches a certain level.

\section{FUEL CONSUMPTION AND EMISSION ANALYSIS}

Besides the enhancement of traffic throughput and safety, fuel consumption and emission reduction is another field where CAVs can make significant contributions. Investigating the fuel efficiency and emission reduction is also an interesting issue in assessing CAVs' contribution to the mixed traffic system. Therefore, this section investigates its potential benefit to energy saving and environment under various CAV market penetration rates and platoon strength based on the simulation results in Section 4, from which each vehicle's kinematic characteristics (instantaneous acceleration and velocity) over simulation time could be obtained. 


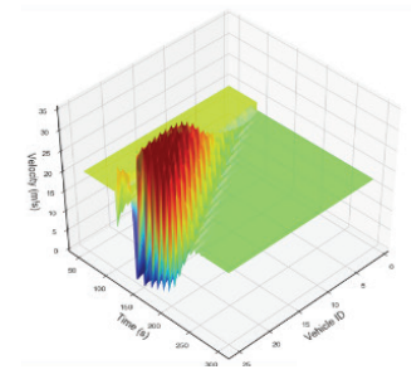

a) $p_{1}=0.1$

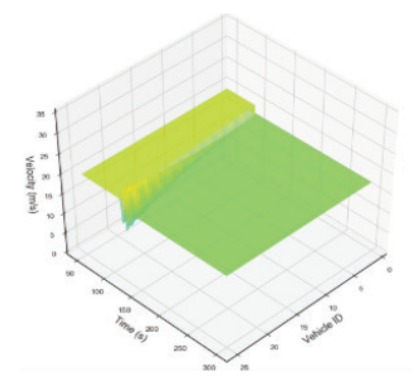

d) $p_{1}=0.6$

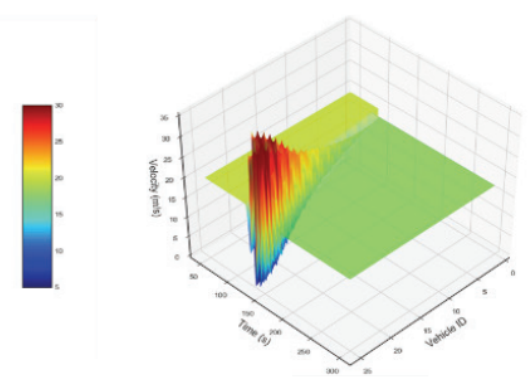

b) $p_{1}=0.3$

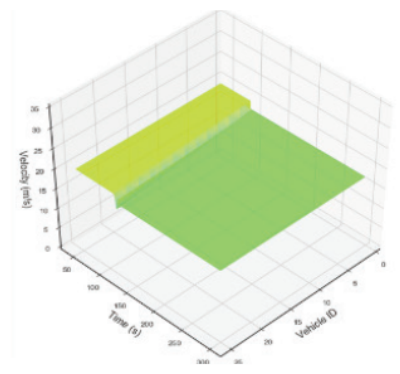

e) $p_{1}=0.9$

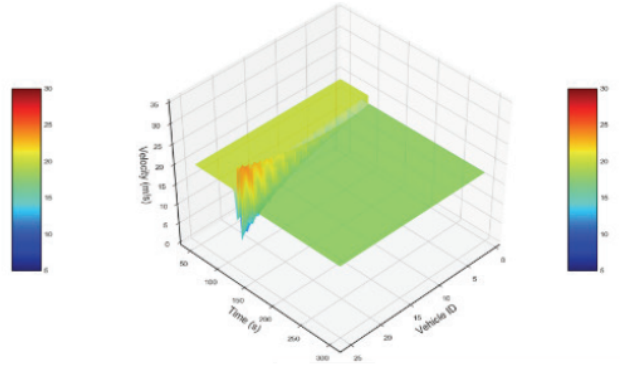

c) $p_{1}=0.5$

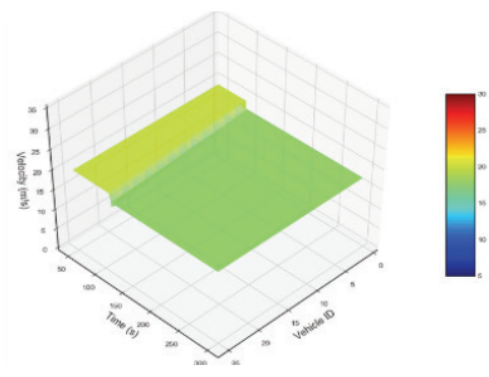

f) $p_{1}=1$

Figure 6 - Propagation of the perturbation along the mixed traffic flow with different CAV market penetrations under open boundary condition

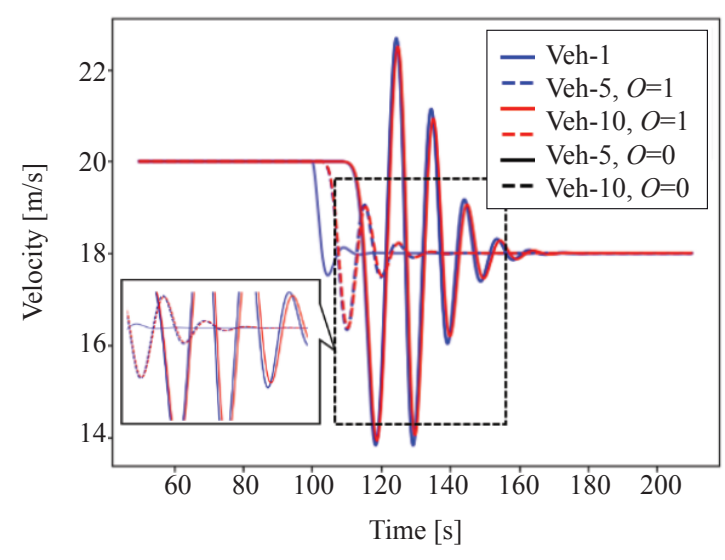

a) $p_{1}=0.1$

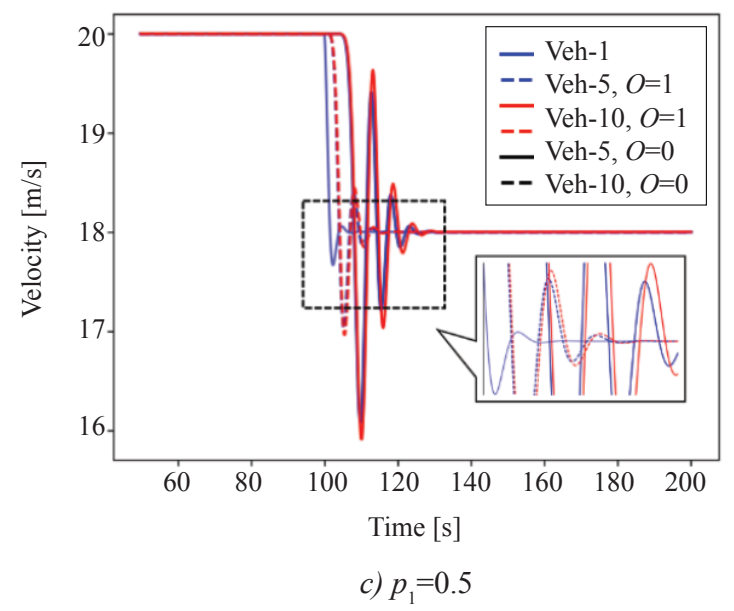

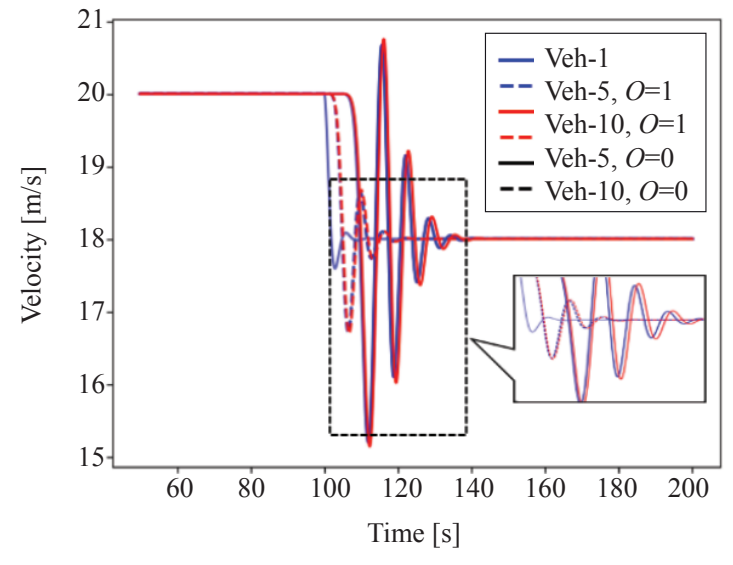

b) $p_{1}=0.3$

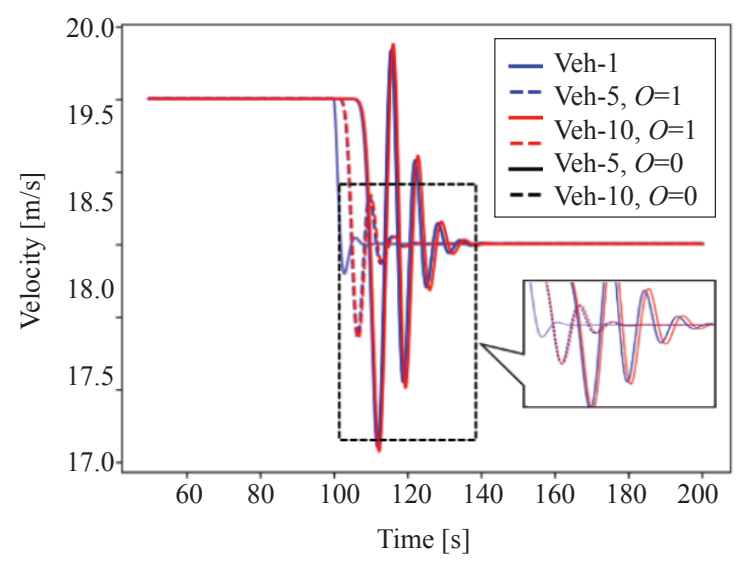

d) $p_{1}=0.8$

Figure 7 - Velocity profiles of some vehicles with different platoon strength of CAVS 
Many scholars have carried out various field experiments of the emissions during the operation of vehicles and obtained some useful empirical formulas [34-36]. For the sake of investigating the environmental influence of vehicles running on a single line freeway segment under a circumstance of different market penetration and platoon strength, this paper adopts the model proposed by Ahn et al. [34]. This is not only because of its good integration with microscopic traffic flow models to explore the contribution of stability performance to exhaust and pollutant emissions, but also for its reasonable estimation accuracy and relatively simple structure, requiring only the vehicle's acceleration and velocity at each moment. Measures of Effectiveness (MOE) indicate the instantaneous fuel consumption $(\mathrm{L} / \mathrm{s})$ of emission rate $(\mathrm{mg} / \mathrm{s})$. For example, for the MOE of fuel consumption, it indicates the instantaneous fuel consumption $(\mathrm{L} / \mathrm{s})$, for the MOE of $\mathrm{CO}_{2}$ emission and $\mathrm{NO}_{\mathrm{x}}$ emission. It represents the instantaneous emission rate $(\mathrm{mg} / \mathrm{s})$. In this model, the fuel consumption or emission rate of $n$-th vehicle MOE can be calculated as the following formulations:

$$
\ln (M O E)=\left\{\begin{array}{l}
\sum_{i=0}^{3} \sum_{j=0}^{3}\left(K_{i, j} \cdot v_{n}^{i} \cdot a_{n}^{j}\right) \text { if } a \geq 0 \\
\sum_{i=0}^{3} \sum_{j=0}^{3}\left(K_{i, j}^{\prime} \cdot v_{n}^{i} \cdot a_{n}^{j}\right) \text { if } a<0
\end{array}\right.
$$

where $v_{n}$ is the velocity of $n$-th vehicle $(\mathrm{km} / \mathrm{h})$; $i=0,1,2,3$ denotes velocity power; $a_{n}$ is the acceleration of $n$-th vehicle $(\mathrm{km} / \mathrm{h} / \mathrm{s}) ; j=0,1,2,3$ represents acceleration intensity; coefficients $K_{i, j}$ and $K_{i, j}^{\prime}$ are both determined by acceleration or deceleration behaviour of the vehicle. In terms of coefficient values for fuel consumption, $\mathrm{CO}_{2}$ emission, $\mathrm{NO}_{\mathrm{x}}$ emission, please see the recommendations in literature [37].
As benefit from the simulation in Section 3, the instantaneous velocity and acceleration could be obtained. Table 4 represents the average reduction of fuel consumption and emission of 25 vehicles with different combinations of market penetration rate and platoon strength, compared with pure HV traffic flow.

For better visualization, Figure 8 illustrates the energy saving and environmental impact with various CAV market penetrations. One can see that the energy and emission reduction deteriorates with the augment of CAV penetration when it is less than about $30 \%$, which is found to be consistent with the results in Figure 7. In addition, it can be easily seen that the platoon strength contributes little to the energy saving and emissions when CAV penetration reaches about $80 \%$. Generally, this figure reveals that substantial potential fuel consumption and emission can be mitigated by more than $100 \%$ under certain scenarios.

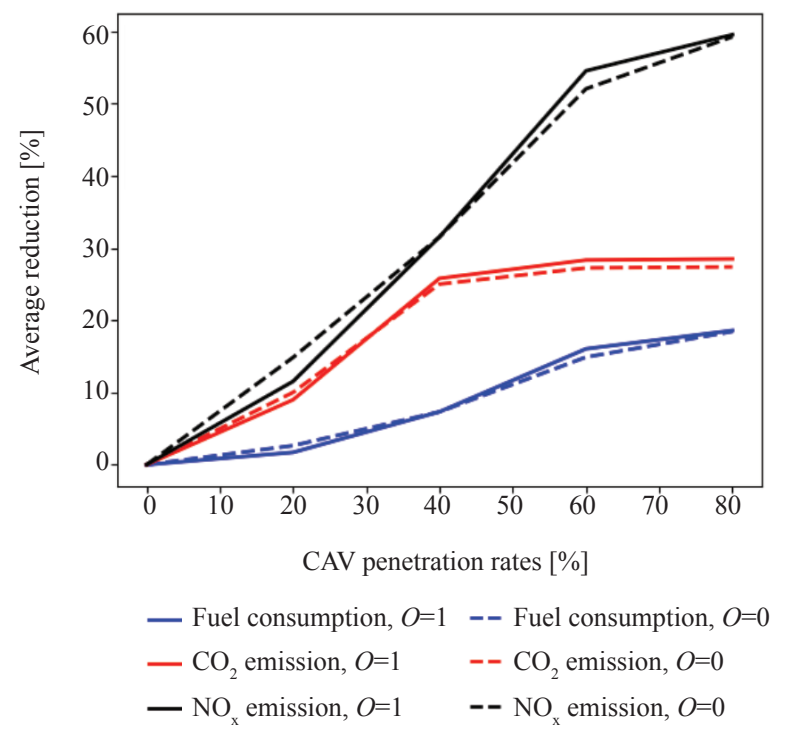

Figure 8-Average reduction of energy and emissions with different $C A V$ market penetration

Table 4 - Average reduction in fuel consumption and emissions

\begin{tabular}{|c|c|c|c|c|c|c||}
\hline \multirow{2}{*}{$\begin{array}{c}\text { CAV market } \\
\text { penetration [\%] }\end{array}$} & \multicolumn{5}{|c||}{ Average reduction } \\
\cline { 2 - 7 } & \multicolumn{2}{|c|}{ Fuel consumption [L/s] } & \multicolumn{2}{c|}{$\mathrm{CO}_{2}$ emission $[\mathrm{g} / \mathrm{s}]$} & \multicolumn{2}{c||}{$\mathrm{NO}_{\mathrm{x}}$ emission $[\mathrm{g} / \mathrm{s}]$} \\
\cline { 2 - 8 } & when $O=1$ & when $O=0$ & when $O=1$ & when $O=0$ & when $O=1$ & when $O=0$ \\
\hline \hline 0 & - & - & - & - & 30.85 & 39.72 \\
\hline 20 & 5.44 & 8.42 & 4.11 & 4.58 & 84.35 & 84.35 \\
\hline 40 & 23.34 & 23.34 & 11.80 & 11.44 & 145.85 & 139.18 \\
\hline 60 & 51.12 & 47.46 & 12.96 & 12.46 & 159.23 & 158.48 \\
\hline 80 & 59.21 & 58.74 & 13.03 & 12.53 & & \\
\hline
\end{tabular}




\section{CONCLUSION}

The main contribution of this paper is to reference an innovative integrated Markov chain-based model to better depict the vehicles' stochastic distribution in the mixed traffic system and derive stability criterion under various sets of CAV penetration rate, CAV platoon strength and collaboration efficiency. This innovative model is found to be useful for linear stability analysis in many cases and can be retrieved into original IDM in specific cases. Consequently, the main novelty and contribution of this paper is the introduction of the concept of platoon strength in the car-following model and stability analysis, taking into account the stochastic probability distribution of different vehicle types in mixed traffic flow by Markov-chain method. The results of numerical analysis and simulations under open boundary conditions show that the innovative integrated model proposed in this paper is a good description of car-following behaviour in a mixed traffic system. Moreover, from the perspective of stability analysis, the contribution of stability performance to energy saving and environmental protection were evaluated, with the results indicating that the substantial potential environmental impact could be reduced under certain scenarios.

Some further extensions of this study need to be carried out in the future. Firstly, linear stability analysis is only the most basic research on the traffic flow. An in-depth study of non-linear analysis deserves more attention. In addition, all the findings in this paper were derived through numerical and simulation analysis due to the lack of field test data of CAVs. Thus, these findings need to be validated by employing the field test data when the real vehicle test site is available. Furthermore, with the development of many other advanced technologies, it should be further investigated how to incorporate more these technologies as relevant factors into our proposed methodological framework. Anyway, we are aware of the stability boundary condition of the mixed traffic system considering the spatial distribution of CAVs and HVs and that advanced technologies (e.g. platoon strength and collaboration efficiency) are able to help smooth the mixed traffic flow by attenuating the perturbation propagation and reduce environmental pollution.

\section{ACKNOWLEDGEMENT}

This research was funded by the National Key R\&D Program in China (Grant No. 2018YFB1600600) and the Postgraduate Research
\& Practice Innovation Program of Jiangsu Province (KYCX19 0108) and the National Natural Science Foundation of China (Grant No. 51878161).

李林恒, 博士研究生 $1,2,3$

电子邮箱: leelinheng@seu.edu.cn

甘婧, 博士研究生 1,2

曲栩, 博士 (通讯作者) $1,2,3$

电子邮箱: quxu@seu.edu.cn

张健, 博士 $1,2,3$

由斌，博士 $1,2,3$

1 东南大学交通学院

中国江苏省南京市江宁区东南大学路 2 号

2 东南大学城市智能交通江苏省重点实验室

中国江苏省南京市

3 东南大学-威斯康星大学智能网联交通联合研究院 中国江苏省南京市

基于马尔科夫链的混合交通流稳定性及环境影 响分析

\section{摘要:}

智能网联汽车市场的快速发展突显了深入了解混 合交通流的整体稳定性的重要性。一些学者研究了 混合交通环境中交通流的建模过程和稳定性分析, 但是缺乏混合交通流内部智能网联汽车及传统汽车 的空间分布考虑。因此, 本文将混合交通流的空间 分布整合到车辆跟驰行为的建模过程中, 建立了一 个新的基于马尔可夫链的跟驰模型。基于此模型, 针对两个跟驰车辆之间不同的合作效率, 智能网联 汽车市场渗透率及链接强度这三个影响因素, 对混 合交通流进行了线性稳定性分析。此外, 为了更清 楚地了解三个因素如何共同影响混合交通流的稳定 性，本文建立多种混合交通流场景，并分别进行了 仿真分析。结果表明, 这三个因素均可以增强混合 交通流稳定性。除了稳定性分析之外，本文还对不 同智能网联汽车市场渗透率及链接强度下的燃油消 耗和污染物排放进行了探索，结果表明在某些场景 下, 智能网联汽车在未来可以大量减少燃油消耗和 污染物排放。

关键词：交通流建模；线性稳定性；智能网联 汽车; 混合交通流; 马尔科夫链

\section{REFERENCES}

[1] Ran B, Cheng Y, Li S, Ding F, Jin J, Chen X, et al. Connected automated vehicle highway systems and methods. Google Patents; 2019.

[2] Wu J, Wang Y, Wang L, Shen Z, Yin C. Consensus-Based Platoon Forming for Connected Autonomous Vehicles. IFAC-PapersOnLine. 2018;51(31): 801-6.

[3] Seraj M, Li J, Qiu Z. Modelling Microscopic Car-Following Strategy of Mixed Traffic to Identify Optimal Platoon Configurations for Multiobjective Decision-Making. Journal of Advanced Transportation. 2018;2018.

[4] Bang S, Ahn S. Platooning strategy for connected and 
autonomous vehicles: Transition from light traffic. Transportation Research Record. 2017;2623(1): 73-81.

[5] Gong S, Du L. Cooperative platoon control for a mixed traffic flow including human drive vehicles and connected and autonomous vehicles. Transportation Research Part B: Methodological. 2018;116: 25-61.

[6] Shi Y, He Q, Huang Z. Capacity Analysis and Cooperative Lane Changing for Connected and Automated Vehicles: Entropy-Based Assessment Method. Transportation Research Record. 2019;0361198119843474.

[7] Virdi N, Grzybowska H, Waller ST, Dixit V. A safety assessment of mixed fleets with Connected and Autonomous Vehicles using the Surrogate Safety Assessment Module. Accident Analysis \& Prevention. 2019;131: 95-111.

[8] Lu C, Dong J, Hu L. Energy-Efficient Adaptive Cruise Control for Electric Connected and Autonomous Vehicles. IEEE Intelligent Transportation Systems Magazine. 2019;11(3): 42-55.

[9] Lu C, Aakre A. A new adaptive cruise control strategy and its stabilization effect on traffic flow. European Transport Research Review. 2018;10(2): 49.

[10] Zhou Y, Wang M, Ahn S. Distributed model predictive control approach for cooperative car-following with guaranteed local and string stability. Transportation Research Part B: Methodological. 2019;128: 69-86.

[11] Qin Y, Wang H, Ran B. Impact of Connected and Automated Vehicles on Passenger Comfort of Traffic Flow with Vehicle-to-vehicle Communications. KSCE Journal of Civil Engineering. 2019;23(2): 821-32.

[12] Wang M. Infrastructure assisted adaptive driving to stabilise heterogeneous vehicle strings. Transportation Research Part C: Emerging Technologies. 2018;91(April 2017): 276-95

[13] Alonso MR, Peralta I, Monti D, Martino R, Anesini C. Stability of an Aqueous Extract of Larrea divaricata Cav. during a Simulated Digestion Process. Phytotherapy Research. 2017;31(11): 1708-14.

[14] Ngoduy D. Analytical studies on the instabilities of heterogeneous intelligent traffic flow. Communications in Nonlinear Science and Numerical Simulation. 2013;18(10): 2699-706. Available from: doi:10.1016/j. cnsns.2013.02.018

[15] Seiler P, Pant A, Hedrick K. Disturbance Propagation in Vehicle Strings. IEEE Transactions on Automatic Control. 2004;49(10): 1835-41.

[16] Ran B, Tsao HJ. Traffic Flow Analysis for An Automated Highway System. $75^{\text {th }}$ TRB Annual Meeting. 1995;(960232).

[17] Ngoduy D. Effect of the car-following combinations on the instability of heterogeneous traffic flow. Transportmetrica B. 2015;3(1): 44-58.

[18] Talebpour A, Mahmassani HS. Influence of connected and autonomous vehicles on traffic flow stability and throughput. Transportation Research Part C: Emerging Technologies. 2016;71: 143-63. Available from: doi:10.1016/j.trc.2016.07.007

[19] Darbha S, Rajagopal KR. Intelligent cruise control systems and traffic flow stability. Transportation Research Part C: Emerging Technologies. 1998;7(6): 329-52.

[20] Gu H, Zhang J, Jin PJ, Ran B. Stability analysis of leadvehicle control model in cooperative adaptive cruise control platoon within heterogeneous traffic flow. Journal of Southeast University. 2018;34: 386-93.

[21] Lu C, Dong J, Hu L, Liu C. An Ecological Adaptive Cruise Control for Mixed Traffic and Its Stabilization Effect. IEEE Access. 2019;7: 81246-56.

[22] Wang J, Peeta S, He X. Multiclass traffic assignment model for mixed traffic flow of human-driven vehicles and connected and autonomous vehicles. Transportation Research Part B: Methodological. 2019;126: 139-68.

[23] Ghiasi A, Li X, Ma J. A mixed traffic speed harmonization model with connected autonomous vehicles. Transportation Research Part C: Emerging Technologies. 2019;104:210-33.

[24] McConky K, Rungta V. Don't pass the automated vehicles!: System level impacts of multi-vehicle CAV control strategies. Transportation Research Part C: Emerging Technologies. 2019;100: 289-305.

[25] Ghiasi A, Hussain O, Sean Z, Li X. A mixed traffic capacity analysis and lane management model for connected automated vehicles: A Markov chain method. Transportation Research Part B. 2017;106: 266-92. Available from: doi:10.1016/j.trb.2017.09.022

[26] Li Z, Li W, Xu S, Qian Y. Stability analysis of an extended intelligent driver model and its simulations under open boundary condition. Physica A: Statistical Mechanics and its Applications. 2015;419: 526-36. Available from: doi:10.1016/j.physa.2014.10.063

[27] Al Alam A, Gattami A, Johansson KH. An experimental study on the fuel reduction potential of heavy duty vehicle platooning. IEEE Conference on Intelligent Transportation Systems, Proceedings, ITSC; 2010. p. 306-11.

[28] Tsugawa S, Kato S, Aoki K. An automated truck platoon for energy saving. 2011 IEEE/RSJ International Conference on Intelligent Robots and Systems; 2011. p. 4109-14. Available from: http://ieeexplore.ieee.org/ document/6094549/

[29] Lammert MP, Duran A, Diez J, Burton K, Nicholson A. Effect of Platooning on Fuel Consumption of Class 8 Vehicles Over a Range of Speeds, Following Distances, and Mass. SAE International Journal of Commercial Vehicles. 2014;7(2): 626-639. Available from: http:// papers.sae.org/2014-01-2438/

[30] Treiber M, Hennecke A, Helbing D. Microscopic simulation of congested traffic. Traffic and Granular Flow '99; 2000. p. 365-76.

[31] Milanés V, Shladover SE. Modelling cooperative and autonomous adaptive cruise control dynamic responses using experimental data. Transportation Research Part C: Emerging Technologies. 2014;48: 285-300.

[32] Wilson RE. Mechanisms for spatio-temporal pattern formation in highway traffic models. Philosophical Transactions of the Royal Society A: Mathematical, Physical and Engineering Sciences. 2008;366(1872): 2017-32.

[33] Ye L, Yamamoto T. Modelling connected and autonomous vehicles in heterogeneous traffic flow. Physica A. 2018;490: 269-77. Available from: doi:10.1016/j.physa.2017.08.015

[34] Ahn K, Rakha H, Trani A, Van Aerde M. Estimating Vehicle Fuel Consumption and Emissions based on Instantaneous Speed and Acceleration Levels. Journal of Transportation Engineering. 2002;128(2): 182-90. 
Available from: doi:10.1061/\%28ASCE $\% 290733-947 \mathrm{X}$ $\% 282002 \% 29128 \% 3 \mathrm{~A} 2 \% 28182 \% 29$

[35] Hausberger S, Rodler J, Sturm P, Rexeis M. Emission factors for heavy-duty vehicles and validation by tunnel measurements. Atmospheric Environment. 2003;37(37): 5237-45.

[36] Barth M, An F, Younglove T, Scora G, Levine C, Ross M, et al. Development of a Comprehensive Modal Emissions
Model. National Cooperative Highway Research Program NCHRP; 2000. Available from: http://onlinepubs. trb.org/onlinepubs/nchrp/nchrp_w122.pdf

[37] Li X, Cui J, An S, Parsafard M. Stop-and-go traffic analysis: Theoretical properties, environmental impacts and oscillation mitigation. Transportation Research Part B: Methodological. 2014;70(1): 319-39. Available from: doi:10.1016/j.trb.2014.09.014 